\title{
CORRECCIONES Y PRECISIONES A VARIOS ESTUDIOS LEXICOGRÁFICOS
}

\author{
Maria Lourdes García Macho
}

Uno de los asuntos que ocupan el tiempo de quienes trabajan con criterios filológicos aplicados al léxico, es el de los errores que se dan en los trabajos de lexicografia, a causa de la interpretación de quienes los confeccionan. Ya que una fecha mal entendida o un lapsus etc. puede dar lugar a serios problemas de consecuencias a veces graves en cuanto la valoración de los datos.

Lo grave sin embargo, es la transmisión de estos errores de obra en obra, por cuanto el trabajo de recopilación lexicográfica se cimenta en el acarreo del material de las obras anteriores.

Es misión del investigador cuando -como ocurre en el presente casose ha acudido a las fuentes ir corrigiendo alguno de esos errores.

Comenzamos indicando los errores que hemos localizado en el $D E C H$, para continuar después con las demás equivocaciones cometidas por otros lingüistas, siguiendo un orden alfabético!

\section{DECH:}

AFECTO:

Infecto (Mena (Smith)): Smith: Pérez de Guzmán.

EFIMERO:

Efeméride (en castellano el sing. efeméride se emplea también en el estilo periodístico, pero no lo autoriza la Acad.): Acad. ya 1970.

EMBAÍR:

(Lamano): Lamano: envair.

\footnotetext{
I Estas pequeñas correcciones fueron recopiladas durante la realización de mi tesis doctoral: Estudio del lenguaje popular y culto de la obra de Luis Maldonado de Guevara y Ocampo. De ahi que en algunas ocasiones se cite a dicho autor.
} 
EMIGRAR:

ÉTICO:

FINGIR:

FLACO:

FONÉTICO:

FUTRE:

LOBO:

LUSTRE:

MICRO-:

ORNAR:

ORQUESTA:

OVACIÓN:
Migratorio (falta Acad. 1939): Ya en Aut., Acad. 1783, 1869 y también en 1939.

(A. de la Torre (Smith)): Smith: Mena. Etica (id.): Smith: A. de la Torre.

Heñir (Nebr.): En Nebr. no figura.

Fláccido (admitido Acad. después de 1899): Acad. ya 1899.

Polifonia (Acad. S. XIX): En la Acad. no figura hasta 1925.

Futraque (hoy desusado según la Acad.): Hasta la ed. de 1956 desusado; sin embargo en la ed. de 1970 ya sin la nota de desusado.

Lupa (rechazado por la Acad.): Acad. ya 1970.

llustrar ('adornar con grabados' Acad. 1869): En la Acad. no figura hasta 1884.

Microbio (Acad. no 1884): Acad. ya 1884 en el apéndice.

Ornamental (Acad. S. XIX): En la Acad. no aparece hasta la ed. de 1936.

Orquestal (falta en la Acad.): Acad. ya 1956.

('aplauso ruidoso' no aparece en Acad. hasta 1914 ó 1899): En la Acad. no figura con esta acepción hasta la ed. de 1925.

PAILA:

Pátina (Acad. 1817): En la Acad. no aparece hasta la ed. de 1832. 
PAMELA:

PAN:

PLANTA:

PREFERIR:

PRIMO:

QUEDO:

QUERER:

RAPIÑA:

REPTIL:

RITO: ('sombrero' Acad. 1914 ó 1899): En la Acad. no figura hasta la ed. de 1925.

Panoli (Acad. 1899 ó 1914): En la Acad. no aparece hasta la ed. de 1936.

Implantar (Acad. S. XIX): En la Acad. no figura hasta la ed. de 1914 'plantear' y en la ed. de 1925 'poner en ejecución doctrinas nuevas, costumbres'.

Delatar (Covarr.): En Covarr. no figura, solamente se encuentra delator.

Principesco (Acad. 1925, no 1843): En la Acad. no aparece hasta la ed. de 1936.

Inquieto (Fz. Villegas (Smith)): En el artículo de Smith este vocablo no figura. Los autores del $D E C H$ han tomado para la voz inquieto, la documentación de la palabra quieto que si aparece en dicho artículo.

Inquisitorial (A. de la Torre (Smith)): En el articulo de Smith esta voz no aparece. Los autores del $D E C H$ han tomado para el vocablo inquisitorial, la documentación del término inquisición, que sí figura en dicho artículo.

Rapto (Covarr.): En Covarr. no aparece.

(Pz. de Guzmán (Smith)): Smith: A. de la Torre.

(APal): no se ha tomado la documentación de Smith: Mena, para esta voz, y en su lugar, se la han colocado equivocadamente al vocablo ritual, el cual no figura en Smith.

TAUMATURGO: Taumatúrgico (Acad. S. XIX): En la Acad. no figura hasta la ed. de 1936.

TORCER: 
Finalmente en la bibliografia que figura en el primer tomo del $D E C H$ se indica que el Vocabulario de las palabras y frases bables de Rato, se publicó en 1892. Sin embargo, esta datación está equivocada, ya que se publicó un año antes en 1891.

ACEVEDO, BABLE:

Senara 'heredad' A. (Vigón): En Vigón, Colunga no figura. Este mismo error está asimismo indicado en el $D E C H$.

ALFARO, ANGLICISMOS:

Folklore: Acad. desde la 14 ed. (1914): En la Acad. no figura hasta la 15 ed. (1925).

D. ALONSO, GÓNGORA:

Hiperbólico, p. 103: Minshew, 1517: Minshew se publicó en 1617.

ALVAR, TEXTOS DIALECTALES:

Aiciones: CCCXXV, 10, arg.: no es el verso 10, sino el 101.

Contino: $\mathrm{CCXCIV}, 15$, and.: $\mathrm{Ni}$ en el verso $15 \mathrm{ni}$ en los siguientes figura este vulgarismo.

BATTANER, POLITICA:

Compromisario: Acad. 1869: Sin embargo con la acepción que figura en el texto que Battaner incluye 'aquel en quien se compromenten los electores para llevar a efecto una elección' no aparece en la Acad. hasta 1899.

Malthusiano: No Acad. 1884: Ya en la Acad. 1884: Maltusiano. 
COVARRUBIAS:

Efemérides: 116, a, 22: Aquí no figura.

Sochantre: 292, b, 35: Aquí no se encuentra.

GARCÍA DE DIEGO, DICCIONARIO:

Esgarrar: ‘desgarrar': Salm. Lamano: En Lamano únicamente tiene las dos acepciones siguientes 'cambiar una moneda por otras de menos valor' y 'toser, arrancando flemas'.

LAIIN, «UNAMUNO»:

Andorga, p. 78: El Dicc. de la Acad. la cita como palabra fam. y vulgar: La Acad. sólo la menciona como voz familiar, en ninguna ed. figura como vulgar.

LAMANO, SALAMANCA (2):

Alcaereño, ña: f. 'lo perteneciente a la alquería', "Casas alcaereñas», L. Maldonado: Funciona como adj., no como sust.

Ameno 'fecundo, abundante', L. Maldonado: En el texto de L. Maldonado significa 'apto, adecuado': «En unos terrenos se dan liebres y otros son amenos de perdices» Elisa, 27.

Amuelar: Aparece un texto sin indicar a quien pertenece: Estos renglones son de L. Maldonado, Cavila: $C$ y $C$., p. 65.

Asin: adj., "Asin como asin»: Es un adv.

Carresquera: Con una cita de L. Maldonado: En este autor únicamente figura carrasquera tanto en la ed. que utiliza Lamano, de 1903, p. 11, como en la de 1932, p. 6, así como en la de 1973, p. 19.

2 Las páginas Del campo y de la ciudad de L. Maldonado, que figuran dentro de estos errores, están citadas de la edición de 1903, debido a que es ésta la edición que utiliza Lamano, cuando se refiere al escritor salmantino. 
Carrilano: adj.: En la Acad. únicamente está atestiguado como sust. y en Maldonado funciona asimismo como sust.

Cualisquiera: Galán, Castellanas, p. 67: Este pronombre no figura en la p. 67, sino en la p. 37.

Cuatropea 'carrera tendida de caballería mayor' Galán, Castellanas, p. 60: Según Z. Vicente, G. y Galán, p. 165 y según el texto que presenta Lamano no tiene esta ac. sino la de 'lugar de una feria donde se vende el ganado'.

Curar: Maldonado, $C . y$ C., p. 87: No es esta pág. sino la 89 y entonces en vez de corresponder al cuento El ama Concención, se trata del cuento titulado Don Lionardo.

Desbruciarse: Aparece un texto sin indicar de quién es: el texto corresponde a L. Maldonado, Recurso: C. y C., p. 110.

Desuñir: Correas, Vocabulario, p. 372: No figura esta voz en esta pág. sino en la pág. 191.

Embuelzas, T. Villarroel, Obras, T. VII, p. 385: En T. Villarroel figura con la grafia envuelzas.

Endentro: Maldonado, Montaraza: En este autor en esta obra únicamente aparece endrento, y se encuentra en la p. 37.

Entenado, da: adj.: La Acad. únicamente recoge este arcaísmo como sust. $\mathrm{m}$. y f. y en L. Maldonado funciona también como sust.

Garlo 'nalsa': 'cajón cuadrado de madera el cual va recogiendo la harina, según van moliendo el grano', 'especie de tinaja para guardar la harina': Pensamos más bien que Lamano queria referirse a 'nasa' que se corresponde con el texto que incluye de Maldonado: "A Juan que eche los garlos a la charca, pa ver si coge un par de libras de tencas».

Jijeo: Maldonado, $C y C$., p. 11 y 141: En L. Maldonado, sin embargo, figura con la grafia jigeo y no solamente en esta ed. de 1903, sino también en la ed. de 1932, p. 6 y 152 y en la de 1973, p. 20 y 124. 
Mañanero: Maldonado, Montaraza, p. 87: No aparece esta voz en esta pág. sino en la p. 27.

Pelra: Maldonado, $C . y$ C., p. 117: no figura en esta pág. sino en la p. 116.

Propetario: adj., Maldonado, C. y C., p. 61: Pensamos que este vulgarismo en este autor funciona como un sust. pl. «Pero como tú no haces leyes, como las hacen los propetarios».

Pupo 'abubillo': En masc. no hemos encontrado registrado este pájaro, se tratará de abubilla: f, forma que si figura en Aut. y en la Acad.

Reñón: Maldonado, $C . y$ C., p. 61: En esa pág. no aparece este vulgarismo sino en la pág. 8.

Tadia: Maldonado, Montaraza, 29: Asimismo en esa pág. no figura este vocablo sino en la pág. 46.

Trillique, ca: adj. 'el niño o niña que dirige la yunta en la trilla': Es un sustantivo.

Tuno, na: adj. 'el que forma parte de la comparsa carnavalesca', «Esta calle abajo va / la vigüela de los tunos»: Tanto en este texto como en L. Maldonado se trata de un sust.

Vergüenza: Dentro de la voz desabuso, Lamano cita un texto de Maldonado y escribe vergüenza: Sin embargo, en este escritor en la ed. de 1903, p. 90 , así como en las eds. de 1932, p. 93 y de 1973, p. 83 figura siempre virgüenza.

\section{LÓPEZ, CUENCA:}

Vacante 'vacaciones': La Acad. lo registra como fam. y arcaico: La Acad. no coloca a este vocablo ningún tipo de nota adicional.

\section{MALDONADO, LUIS, ANTOLOGÍA:}

La Velada de Galán: Publicada en 1920: No fue publicada en ese año, sino en 1922. 
La Golisa de Alizán: Novela premiada en el año 1900: no fue premiada en ese año, sino en el año 1902.

MALDONADO, LUIS, C. y C., ed. 1903:

Le concejo, p. 60: Sin embargo lo correcto es $E$ l concejo, falta ya subsanada en la ed. de 1932, p. 61 y en la ed. de 1973, p. 59.

Gracis, p. 142: En lugar de la forma correcta gracias, la cual ya figura en las eds. de 1932, p. 153 y de 1973, p. 125.

Uso, p. 45: Cuando deberia decir huso. Voz que ya se encuentra corregida en las eds. de 1932, p. 46 y de 1973, p. 48.

MALDONADO, LUIS, C. $y$ C., ed. 1932:

Cabo, p. 183: Cuando debería decir cavo, ya que es una forma del verbo cavar, la cual ya está corregida en la ed. de 1973, p. 151.

Coorte, p. 221: Sin embargo ya figura cohorte en la ed. original publicada en el "Lábaro", y en la ed. de 1973, p. 181.

Exámine, p. 22 y el mismo error en la ed. de 1973, p. 30: Sin embargo, la forma correcta exánime ya aparece en la publicación original y en la ed. de 1903, p. 23.

MALDONADO, LUIS, $C, y$ C., ed. 1973:

Azapón, p. 59: Cuando debería decir alzapón como ya figuraba en la ed. de 1903 , p. 60 y en la ed. de 1932, p. 61.

Exámine, p. 30: Por exánime como acabamos de indicar.

Metistofélica, p. 116: Cuando la forma correcta es mefistofélica, como aparece en las eds. de 1903, p. 128 y de 1932, p. 139. 
Queria, p. 93: sing.: Sin embargo debería decir querian (ya que se está refiriendo a los mambises), como está ya correctamente escrito en las eds. de 1903, p. 102 y de 1932, p. 107.

MARCOS, BÉEJA:

Antier:Z. Vicente: Marcos señala que este vocablo figura en $Z$. Vicente, sin especificar en cual de los diversos estudios de este autor aparece. Hecho por otra parte que debia hacer constar, puesto que por poner un ejemplo, en su estudio sobre G. y Galán, p. 147 lo que encontramos es antiayel y es en su trabajo sobre Mérida, p. 50 donde aparece registrado antier.

Apensar: L. Maldonado: En este autor figura apiensar.

Arrecadar: En Llorente: En Llorente, Ribera, p. 179 únicamente figura recadar. Este error, Marcos lo tomó de Sánchez, "Medina del Campo" que dice lo mismo.

Burriciego: Lamano: En Lamano lo único que figura es burriciégano.

Carear: Marcos y además Alcalá, Andaluz y García Cabañas, Alpujarra, señalan que esa voz según el $D R A E$ 'pacer el ganado' como de Salm. Sin embargo, no indican que las eds. de la Acad. en las que esto se dice son las de 1925, 1936 y 1947. Asimismo Marcos indica que la 5 ac. 'pacer' y la 7 ac. 'oxear' como de Salm, en la Acad. Teniendo en cuenta que este vocabulario de Béjar se publicó en 1979, debería el autor especificar que se trata de la ed. de 1925, puesto que en la ed. de 1970, la 3 ac. corresponde a 'pacer' y viene ya sin indicación de Salm. y la 6 ac. 'oxear' sigue con la nota de Salm., pero ha variado el $n .^{\circ}$ de la ac.

Comuelgo: Dentro del vocablo cogolmo, señala comuelgo en Z. Viente, $G$. $y$ Galán, p. 168: No figura en esta pág. sino en la pág. 169.

Chacho: Garrote, p. 155: Sin embargo, en Garrote no figura en esa pág., ni en el vocabulario que nos ofrece este libro, sino en la p. 93 dentro de las figuras de dicción: aféresis. Este mismo error figura en $\mathrm{Z}$. Vicente, $M \dot{e}^{-}$ rida. 
Cheira: Lamano y Acevedo como 'instrumentos de hierro para afilar las cuchillas': En Acevedo es chaira, y no cheira, ya que esto último en este libro significa 'llanura'.

Rolla: DRAE como propio de Badaj., Vallad., Colomb., León, Palenc. y Zam.: Se debería indicar que con estas indicaciones figura en las eds. de la Acad. de 1925 y de 1947, puesto que en la ed. de 1970 no aparecen señaladas ninguna de estas ciudades.

MARTY, VOCES QUE FALTAN EN LA ED. ACAD. 1852:

Licenciatura: No figura Acad. 1852: Sin embargo, esta voz ya se encuentra en esa ed.

Tricornio: No aparece Acad. 1852: Si está registrada en la ed. de 1852 de la Acad.

M. MOLINER, DICCIONARIO:

Rictus: No mencionado en el $D R A E$ : Sin embargo, este vocablo ya figura en el apéndice de la ed. de la Acad. de 1947.

QUILIS, «ALBACETE»:

Mercar 'comprar' en Borao: En Borao no figura esta voz.

REAL ACADEMIA, DICCIONARIO, ED. 1925:

Perlada: adj. 'perlina' igual figura en el $D E C H$ : sin embargo, deberian haber puesto la forma masc., puesto que de los adjs. lo normal es poner el masc. y el fem. como ya aparece en la ed. de la Acad. de 1970.

Terraguero: V. Terrero, 11 ac.: La 11 a. es 'plaza pública', 10 cual quiere decir que no se trata de la 11 ac., sino de la 12 ac. que dice 'montón que en la era se forma con las barreduras del solar de la parva' que es precisamente lo que significa terraguero. 
REAL ACADEMIA, DICCIONARIO, ED. 1970:

Cuqueria 'taimeria': Es taimaria.

Verderón: con la nota de Al.: En lugar de poner Sal., como figura en la ed. de la Acad. de 1925. Además este vocablo es un salmantinismo.

RIVODO, VOCES NUEVAS:

Colectividad: Falta en la duodécima ed. de la Acad. (1884): sin embargo, esta voz ya está registrada en el apéndice de esta ed. de 1884.

G. SALVADOR, «CÚLLAR» (1957), p. 248:

$\mathrm{R}=\mathrm{L}$ : Blincar. Del cambio contrario $\mathrm{R}=\mathrm{L}:$ Brusa: Debería decir $\mathrm{L}=\mathrm{R}$ : Brusa.

SÁNCHEZ, «MEDINA DEL CAMPO» p. 241:

Arrecadar: Llorente: En Llorente, Ribera, p. 179, únicamente figura recadar.

TRINIDAD, MADRID:

Agüelos: Acad ya 18 ed. (1956): Ya figura en Covarr., Aut...

Arrematar: Acad. ya 18 ed. (1956): Acad. ya 1914...

Cuido: Acad. ya 18 ed. (1956): Acad. ya 1869...

Fachenda: Acad. ya 18 ed. (1956): Acad. ya 1817...

Gachó: Acad. ya 18 ed. (1956): Acad. ya 1925...

Guasa: Acad. ya 18 ed. (1956): Acad. ya $1869 \ldots$ 
Guindilla: Acad. ya 18 ed. (1956): Acad. ya 1914...

Morral: Acad. ya 18 ed. (1956): Acad. ya 1869...

Panoli: Acad. ya 18 ed. (1956): Acad. ya $1936 \ldots$

Sofoco: Acad. ya 18 ed. (1956): Acad. ya 1884...

ZAMORA VICENTE, MÉRIDA:

Chacho: Garrote, p. 155: no figura en esta pág. sino en la pág. 93.

ZAMORA VICENTE, G. Y GALÁN:

Arraseas: p. 157: En esta pág. ese vocablo no figura, se referirá a arrascas, que es la voz que aparece en esa pág.

Remudo: Figura en el Dicc. académico: En las diferentes eds. de la Acad. no figura, únicamente se encuentra registrado en Terreros. 


\section{BIBLIOGRAFIA}

Acevedo, B. y Fernández, M., Vocabulario del bable de occidente, S. Aguirre, Impresor, Madrid, 1932.

Alfaro, R.J., Diccionario de anglicismos, Gredos, Madrid, 1964.

Alonso, D., La lengua poética de Góngora, 2." ed., Anejo XX de la RFE, Madrid, 1950.

Alvar, M., Textos hispanicos dialectales: Antologia histórica, 2 tomos, C.S.I.C., Anejo LXXIII de la RFE, Selecciones Gráficas, Madrid, 1960.

BatTaner, M.P., Vocabulario politico-social en España (1868-1873), Anejo XXXVII del BRAE, Madrid, 1977.

COROMInAS, J. y PASCUAL, J. A., Diccionario critico etimológico castellano e hispánico, 5 tomos, Gredos, Madrid, 1980-1983.

Covarrubias, S., Tesoro de la lengua castellana o española (1611, 1674), ed. de Martín de Riquer, S. A. Horta, Barcelona, 1943.

Garcia de Ditgo, V., Diccionario etimológico español hispánico, S.A.E.T.A., Madrid, 1954

LAIN, M., "Aspectos estilisticos y semánticos del vocabulario poético de Unamuno", Cuadernos de la Cátedra Miguel de Unamuno, IX, 1959, pp. 77-115.

Lamano y BeneITE J., El dialecio vulgar salmantino. Tipografia Popular, Imp. de "El Salmantino», Salamanca, 1915.

LOPEZ BARRERA, J., Estudios de semántica regional. Arcaismos y barbarismos de la provincia de Cuenca, Imp. sucesor de J. Gómez Madina, Cuenca, 1912.

Maldonado de Guevara, L., Antologia de las obras de D. Luis Maldonado. Imp. Ferreira, Salamanca, 1928.

- Del Campoy de la ciudad, Imp. y Lib. de Francisco Núñez, Salamanca, 1903.

- Del Campo y de la ciudad, 2." ed., Imp. de Calatrava, Salamanca, 1932.

- Del campo y de la ciudad, 3." ed., Gráficas Cervantes, Salamanca, 1973.

Marcos Casquero, M.A., El habla de Béjar: Léxico, Gráficas Europa, Salamanca, 1979.

MARTY, L., Vocabulario de todas las voces que faltan a los diccionarios de la lengua castellana. Imp. de Anselmo Sta. Coloma, Madrid, 1859.

Moliner, M., Diccionario de uso del español, Gredos, 2 tomos, Madrid, 1982.

Quilis Morales, A., "El habla de Albacete", RDTP, XVI, 1960, pp. 413-442.

Real ACADEmia Española, Diccionario de la lengua española, 15." ed., Talleres Calpe, Madrid, 1925.

Real ACademia Española, Diccionario de la lengua española, 19." ed., EspasaCalpe, Madrid, 1970.

Rivodo, B., Voces nuevas en la lengua castellana, Lib. española de Garnier, Paris, 1889.

Salvador, G., "El habla de Cúllar-Baza", RFE, XLI, 1957, pp. 161-252.

SANCHEZ LOPEZ. L., "Vocabulario de la comarca de Medina del Campo", RDTP, XXII, 1966, pp. 239-303.

TRINIDAD, F., Un estudio del habla popular madrileña, Ed. Góngora, Madrid, 1969.

Zamora Vicente, A., El habla de Mérida y sus cercanias, Anejo XXIX de la RFE,

S. Aguirre impresor, Madrid, 1943.

- El dialecialismo de José $M .^{a}$ Gabriel y Galán, Instituto de Filología Románica, Buenos Aires, 1950. 